\title{
A Diffusion Model Account of Age Differences in Posterror Slowing
}

\author{
Gilles Dutilh \\ University of Amsterdam and University of Basel
}

\author{
Birte U. Forstmann \\ University of Amsterdam and Max Planck Institute for Human \\ Cognitive and Brain Sciences, Leipzig, Germany
}

\author{
Joachim Vandekerckhove \\ Leuven University
}

Eric-Jan Wagenmakers
University of Amsterdam

\begin{abstract}
People generally slow down after they make an error, a phenomenon that is more pronounced for older individuals than it is for young individuals. Here, we examine the origin of this age-related difference in posterror slowing (PES) by applying the diffusion model to data from young and older participants performing a random dot motion task and a lexical decision task. Results show that the PES effects on response time and accuracy were qualitatively different for young and older participants. A diffusion model analysis revealed that following an error, older participants became more cautious, processed information less effectively, and spent more time on irrelevant processes. This pattern was evident in both the random dot motion task and the lexical decision task. For young participants, in contrast, the origin of the PES effect depended on the task that was performed: In the random dot motion task, the PES effect was due to time spent on irrelevant processes; in the lexical decision task, the PES effect was due to increased caution and decreased effectiveness in information processing. Overall, PES effects were much larger in the lexical decision task than in the random dot motion task. These findings indicate that PES originates from the interplay of different psychological processes whose contribution depends on both task settings and individual differences.
\end{abstract}

Keywords: response times, diffusion model, post-error slowing, aging

In most cognitive tasks, older people respond more slowly than young people (Salthouse, 1985). Early research has led to the pessimistic claim that older people slow down because they suffer from a general decrease in the efficiency with which neurons transfer information (Brinley, 1965; Cerella, 1985). Today, however, many researchers agree that older people slow down, at least in part, because they choose to be more cautious than young people. This means that older participants often choose to collect more evidence before they are willing to commit to a decision, a strategy that may result in a substantial loss of speed in return for a small gain in accuracy (Ratcliff,

This article was published Online First September 3, 2012.

Gilles Dutilh, Department of Psychology, University of Amsterdam, Amsterdam, the Netherlands; Department of Psychology, University of Basel, Basel, Switzerland; Birte U. Forstmann, Department of Psychology, University of Amsterdam, Amsterdam, the Netherlands; Max Planck Institute for Human Cognitive and Brain Sciences, Leipzig, Germany; Joachim Vandekerckhove, Faculty of Psychology and Educational Sciences, Leuven University, Leuven, Belgium; Eric-Jan Wagenmakers, Department of Psychology, University of Amsterdam, Amsterdam, the Netherlands.

This research was supported by Veni and Vidi grants from the Dutch Organization for Scientific Research. We thank Roger Ratcliff for providing us with the data that are analyzed in Experiment 2.

Correspondence concerning this article should be addressed to Gilles Dutilh, University of Basel, Department of Psychology, Missionsstrasse 60/62, 4055 Basel, Switzerland. E-mail: gilles.dutilh@gmail.com
Thapar, \& McKoon, 2006b, 2010; Salthouse, 1979; Starns \& Ratcliff, 2010; Strayer, Wickens, \& Braune, 1987; but see Mata \& Nunes, 2010).

Older people are not just more cautious than young people; they also appear to have coarser control over their speed-accuracy tradeoff (Band \& Kok, 2000; Rabbitt, 1979; Smith \& Brewer, 1995). This relatively coarse cognitive control is thought to be reflected in more pronounced posterror slowing (PES). Posterror slowing is the phenomenon that participants, after committing an error, tend to slow down on the next trial. The common explanation of PES states that participants constantly monitor their performance and that an error signals the need for more cognitive control in order to keep performance at an acceptable and relatively constant level of accuracy. Specifically, participants are thought to interpret an error as a sign that response criteria need to be increased; this increase ensures that the decision on the next trial is based on more information, reducing the probability of a second consecutive error. Thus, the fact that older participants show a relatively pronounced PES effect may be due to an excessive increase in response criteria following an error. This explanation is conceptually consistent with the hypothesis that older individuals are more cautious because they are highly motivated to avoid errors.

However, other explanations of PES have been proposed. For instance, PES could also be the result of a distraction of attention (Notebaert et al., 2009) or delayed startup of information processing due to time spent on irrelevant processes (e.g., overcoming disappointment; Rabbitt \& Rodgers, 1977; for a recent review, see Danielmeier \& Ullsperger, 2011). 
In this study, we assessed the psychological processes responsible for the difference in PES between older and young participants. To do so, we needed to overcome two obstacles that complicate the analysis of PES. First, speed and accuracy are in trade-off and only simultaneous analysis of both variables can reveal the psychological processes that underlie behavior. Therefore, in this study, we applied the Ratcliff diffusion model (Ratcliff, 1978; Ratcliff \& Tuerlinckx, 2002) to our data. The diffusion model allows one to take into account response time (RT) and accuracy simultaneously and to decompose the effects on both variables in psychologically relevant underlying constructs. A second challenge is pointed out by Dutilh, van Ravenzwaaij, et al. (2012), who showed that the standard method of quantifying PES (i.e., calculating the difference $\overline{R T}_{\text {posterror }}-\overline{R T}_{\text {postcorrect }}$ ) is confounded with global changes in performance over the course of an experiment; these changes can create spurious PES or mask real PES. Therefore, we applied the solution that is described by Dutilh et al. and quantified PES by a local "robust" measure, that is, by the difference in RT prior to and following each individual error.

The outline of this article is as follows. First, we shortly discuss the literature on aging effects in RT tasks and the role of posterror slowing. Second, we introduce the diffusion model. The third section describes how PES analyses can be confounded by global changes in behavior. Next, we present data from an experiment that manipulates the speed-accuracy trade-off in a random dot motion task. We present the results in terms of RT and accuracy and in terms of the diffusion model parameters. While presenting the results, we illustrate the importance of using the method described by Dutilh, van Ravenzwaaij, et al. (2012) to overcome confounds of global changes in performance. Finally, we assess the generalizability of our results by applying the same PES analyses to an existing data set on lexical decision (Ratcliff, Thapar, \& McKoon, 2004).

\section{Response Speed and Age}

For many years, the difference in response speed between young and older participants has been taken as support for a generalizedslowing hypothesis of aging. This hypothesis states that people slow down with age because all processes in the brain deteriorate (Brinley, 1965; Salthouse, 1996).

Relatively recently, this generalized-slowing hypothesis was challenged by Ratcliff and colleagues in a series of articles in which these researchers used the drift diffusion model to obtain a more detailed assessment of the effects of aging on task performance (e.g., Ratcliff, Spieler, \& McKoon, 2000; Ratcliff et al., 2004; Ratcliff, Thapar, \& McKoon, 2006a; Ratcliff et al., 2006b, 2010; Thapar, Ratcliff, \& McKoon, 2003). As we show below, the diffusion model allows one to decompose effects on RT and accuracy into unobserved psychological processes. Diffusion model decompositions showed that older participants were almost always slower than young participants in the nondecisional components of RT (e.g., stimulus encoding and motor time). The diffusion model decompositions also showed that in many tasks, older participants responded more cautiously than young participants (see also Strayer et al., 1987). It is important to note that only in a subset of tasks (i.e., perceptual decision-making tasks) did the diffusion model decompositions suggest that older participants were less effective than younger participants in extracting information from the stimulus (but see Spaniol, Madden, \& Voss,
2006). These findings suggest that the general-slowing hypothesis of aging is problematic. Instead, detailed diffusion model analyses suggest that age-related slowing is a multifaceted phenomenon that is often dominated by processes unrelated to the effectiveness with which information is extracted and accumulated.

Older participants not only respond more slowly than young participants on average, but they also exhibit a more pronounced PES effect (Band \& Kok, 2000; Rabbitt, 1979; Rabbitt \& Vyas, 1980; Smith \& Brewer, 1995). It is tempting to conclude that, following an error, older participants increase their response thresholds more than young participants (Smith \& Brewer, 1995). However, there are several alternative explanations for how an error influences performance on the next trial, such as (a) the error distracts attention away from the task (Notebaert et al., 2009); (b) the error shifts people's a priori bias away from the response that was executed in error (Laming, 1968; Rabbitt \& Rodgers, 1977); (c) the error delays the startup of information accumulation because time is spent on irrelevant processes such as overcoming disappointment (Rabbitt \& Rodgers, 1977); or (d) the error causes people to time the onset of the stimulus more precisely, preventing the sampling of irrelevant information (Laming, 1968, 1979a).

Dutilh, Vandekerckhove, Forstmann, and Wagenmakers (2012) have shown that the diffusion model is able to discriminate between the alternative explanations of PES listed above (see also White, Ratcliff, Vasey, \& McKoon, 2010). In this study, we used the diffusion model to assess whether the PES effect in young and older participants originates from changes in the same or different psychological processes.

\section{Accounting for Speed and Accuracy Simultaneously}

There is a trade-off relation between RT and accuracy, and this trade-off compromises the analysis of task performance when RT and accuracy are considered in isolation (e.g., Wagenmakers, Van der Maas, \& Grasman, 2007; Wickelgren, 1977). In particular, it can be difficult to assess performance differences between young and older participants when the latter are known to respond more cautiously, accepting a large loss in RT for small gains in accuracy. To quantify performance differences in the face of the speed-accuracy trade-off, one requires a formal model of information processing. In this study, we applied the diffusion model. The diffusion model decomposes effects on RT and accuracy into effects on underlying psychologically relevant processes. The diffusion model naturally accounts for the speed-accuracy trade-off, a key feature for the analysis of age-related slowing.

\section{The Diffusion Model}

In the diffusion model for speeded two-choice tasks (Ratcliff, 1978), stimulus processing is modeled as the noisy accumulation of evidence over time. When the accumulated evidence reaches a predefined evidence boundary, a response is initiated (see Figure 1). The four main parameters of the diffusion model are (1) drift rate $v$ (drift rate quantifies the speed of information processing, reflecting the difficulty of the stimulus and the ability of the participant); (2) boundary separation $a$ (boundary separation quantifies response caution, and thus determines the speed-accuracy tradeoff); (3) starting point $z$ (starting point quantifies a priori bias for one the response options; and (4) nondecision time $T_{e r}$ (nondeci- 

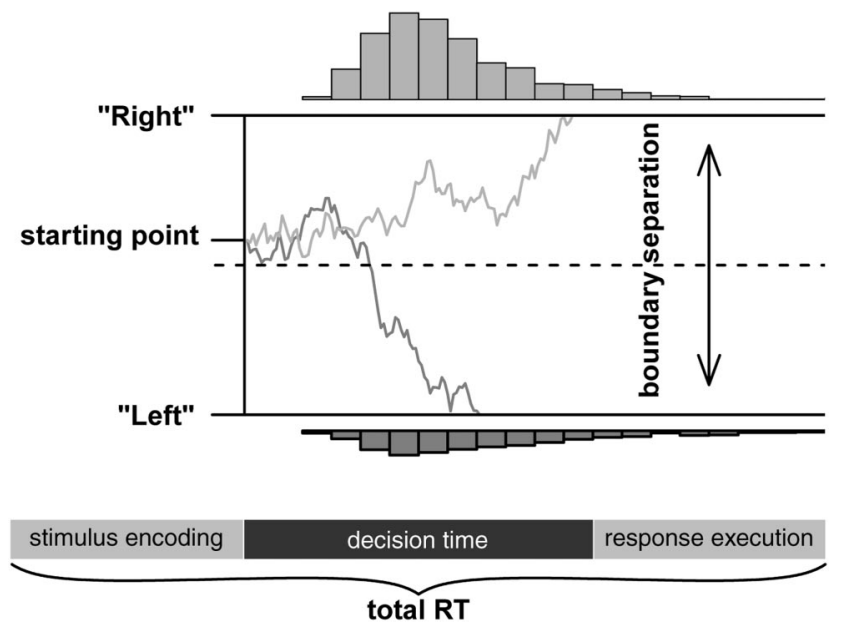

Figure 1. Graphical illustration of the diffusion model. A "Right" stimulus is presented (in this study, random dots motion to the right; see Method section of Experiment 1). The two example sample paths represent the accumulation of evidence that results in one correct response ("Right," light line) and one error response ("Left," dark line). Repeated application of the diffusion process yields histograms of both correct responses (upper histogram) and incorrect responses (lower histogram). As is evident from the histograms, the correct, upper boundary is reached more often than the incorrect, lower boundary. The total response time (RT) consists of the sum of a decision component, modeled by the noisy accumulation of evidence, and a nondecision component that represents the time needed for processes such as stimulus encoding and response execution.

sion time quantifies the time used for encoding the stimulus and executing the motor response). The model specification is completed by including parameters that specify across-trial variabilities in drift rate, starting point, and nondecision time (Ratcliff \& Tuerlinckx, 2002). These variabilities allow the model to account for empirical phenomena such as situations in which errors are systematically faster or slower than correct responses. For a more elaborate introduction to the diffusion model and a review of applications, see Ratcliff and McKoon (2008) and Wagenmakers (2009).

The preferred method to analyze RT data is to use the full diffusion model as described above. However, to fit the diffusion model, one needs at least enough data to estimate the distribution of correct RTs and, more problematic, a distribution of at least, say, 10 error RTs. Because the crucial condition in this study consisted of trials that followed an error, we needed at least 10 errors that immediately followed errors. For this reason, it was not possible to fit the full diffusion to data of individual participants in this study.

\section{The EZ-Diffusion Model}

Fortunately, we could still apply the EZ-diffusion model (Wagenmakers et al., 2007) to the data. A crucial advantage of this simplified version of the diffusion model is that one can calculate the model parameters in closed form from the percentage correct, the RT variance, and the RT mean. In contrast to the full model, this model uses only the parameters drift rate $v$, boundary separation $a$, and nondecision time $T_{e r}$ Thus, the model lacks the variability parameters and bias parameter $z$. Nonetheless, van Ravenzwaaij and Oberauer (2009) have show that the EZ-diffusion model is capable of capturing experimental effects in its parameters and that the model is especially useful when studying individual participants. For other applications of the EZ-diffusion model, see, for example, Schmiedek, Oberauer, Wilhelm, Suss, and Wittmann (2007), Schmiedek, Lövdén, and Lindenberger (2009), Kamienkowski, Pashler, Dehaene, and Sigman (2011), and van Ravenzwaaij, Dutilh, and Wagenmakers (2012).

The EZ-diffusion model allowed us to test three different explanations of PES for this data set: (1) An error distracts attention from the stimulus (PES effect in $v$ ), (2) an error leads to an increase in response caution (PES effect in $a$ ), and (3) an error delays the startup of information processing due to time spent on irrelevant processes (PES effect in $T_{e r}$ ). The use of the EZdiffusion model did not allow us to test posterror effects on a priori bias for one of the response options, as quantified by $z$ in the full diffusion model. Note that the different explanations of PES are not mutually exclusive, and behavior can be affected by more than one of these processes simultaneously.

\section{Quantification of PES}

The most obvious and popular way to quantify PES is to compare trials that follow errors with trials that follow correct responses. However, Dutilh, van Ravenzwaaij, et al. (2012; see also Laming, 1979b, p. 205) showed that this traditional analysis of PES is vulnerable to a confound of global changes in behavior over the course of an experiment. The confound can be best understood by considering the following hypothetical scenario. A participant starts an experimental session highly motivated. The participant is very focused and responds quickly and accurately. Over the course of the experimental session, however, fatigue starts to kick in and consequently responses become slower and less accurate. Now, consider that this participant does not slow down after errors, that is, there is no true PES effect whatsoever. When calculating the difference between RT on posterror trials versus postcorrect trials, most posterror observations originate from the last part of the session when the participant was least motivated and most errors occurred. In that part of the session, the participant responded slowly overall, resulting in a relatively slow mean RT for posterror trials. In contrast, most postcorrect trials originate from the first part of the session when accuracy was high and RT low, yielding a relatively low mean RT on postcorrect trials. Thus, the comparison of posterror versus postcorrect trials will indicate a PES effect, although real posterror slowing is absent. Dutilh, van Ravenzwaaij, et al. (2012) show that, in addition to spuriously detecting PES, the traditional analysis can also underestimate or mask real PES.

The solution offered by Dutilh, van Ravenzwaaij, et al. (2012) is simple: Compare posterror trials with those postcorrect trials that are also pre-error trials. This extra condition assures that trials that comprise both sides of the comparison originate from the same locations in the data set: surrounding errors. For convenience, we refer to the conditions in this robust comparison as posterror versus pre-error.

\section{Experiment 1: Random Dot Motion}

We first describe the method and results of a random dot motion experiment. Random dot motion is a relatively low-level perceptual discrimination task with modest levels of error awareness. 
Later, we assess the generalizability of our findings by comparing the results from the random dot motion task with those from a more high-level task, lexical decision (i.e., Experiment 2).

\section{Method}

Participants. We tested 15 young people (student age; $M=$ 21.80 years, $S D=2.27$ ) and 19 older people $(60-80$ years old; $M=68.27$ years, $S D=5.89$ ) on two occasions within a time span of 2 weeks. Older participants were recruited from the Seniorlab database. ${ }^{1}$ The young participants were rewarded by course credits. The older people participated for a monetary reward.

Individualized deadlines. In the experiment, we alternated blocks that required participants to respond accurately with blocks that required participants to respond quickly. In the accuracy blocks, participants were told to respond accurately but not waste time. In the speed blocks, participants were asked to respond quickly and risk committing errors. To encourage fast responding in the speed blocks, we used a deadline. When participants took longer than the deadline to respond, they received the warning message "too slow" on the computer screen.

As mentioned above, recent studies suggest that older participants have longer nondecision times than younger participants (Ratcliff et al., 2006b; Thapar et al., 2003). Therefore, it was unreasonable to confront participants of both ages with the same deadline. In the diffusion model, nondecision time is related to total RT as follows:

$$
R T=\text { decision time }+T_{e r}
$$

This equation shows that with the same deadline on RT, older participants-who have relatively long nondecision times $T_{e r}$ - have available less decision time than young participants. Because we wanted young and older participants to experience a comparable level of speed stress, we adjusted the deadline for each participant individually and computed it as follows:

$$
R T_{\text {deadline }}^{i}=\text { decision time }+T_{e r}^{i},
$$

in which $R T_{\text {deadline }}^{i}$ is the individualized deadline for participant $i$. This personal deadline was computed by adding participant $i$ 's personal estimate of $T_{e r}$ to a fixed decision time that was the same for all participants. Each participant's personal estimate of $T_{e r}$ was calculated at the start of the experiment. This procedure permits similar decision times for participants with varying nondecision times.

Materials. Participants completed a random dots motion task (Britten, Shadlen, Newsome, \& Movshon, 1992). The random dots motion stimulus consists of a circular display of dots. The dots appear, disappear, and are replaced in such a way that the entire circle of dots appears to move. The apparent motion that the participant perceives can be best described as the flickering of a turning disco ball in a spotlight. This illusion is created as follows. At each frame (50 ms), 120 dots are displayed. Every next frame, an experimentally defined proportion $P_{\text {move }}$ of the dots from the former frame are shifted a certain distance $l_{\text {move }}$ to the target side (e.g., right, if the correct response is right). The remaining portion of the pixels is randomly replaced in the circle (independent of their previous positions). $P_{\text {move }}$ was set to $50 \%$; $l_{\text {move }}$ was set to 1 pixel. We choose a dot size that is bigger than normal (diameter of 4 pixels) to account for the fact that some of the older participants might have had small visual impairments. The circular aperture had a 13-cm diameter. Presentation (Version 09.24.07) for Windows was used to present the stimuli and register the responses. Participants responded to the stimuli by pressing one of two response buttons to indicate the direction of the apparent movement (left or right).

Procedure. In two sessions within 2 weeks, participants were tested individually in a room in which the experimenter was present during the entire experiment. For each participant, the two sessions took $1 \mathrm{hr}$ each.

The first session started with two 50-trial training blocks. One training block had speed instructions. In this block, a "too slow" feedback was displayed when a deadline of $650 \mathrm{~ms}$ was passed. The other training block had accuracy instructions and an "incorrect" feedback message was given on incorrect responses. Also, after 1,500 ms, a "no response" warning was shown.

After participants were trained on the stimuli and the deadline in the speed blocks, another 300-trial speed block was administered. The data of the last 250 trials of this block were used for the personal estimate of $T_{e r}$ This personal estimate of $T_{e r}$ was then used to calculate the personal deadline, as described above. Based on a pilot study with the same task, we choose $R T_{\text {decision }}=200 \mathrm{~ms}$ because this value plus an average $T_{e r}$ yielded a reasonable deadline. The personal deadline that was determined with this procedure was used in the speed blocks throughout both sessions of the experiment.

After the practice blocks and the block to calculate the nondecision time estimate, the main experiment started. Blocks with speed instructions were alternated with blocks with accuracy instructions. Each block contained 100 trials. After each block, participants had a self-paced break. Testing was limited to $2 \mathrm{hr}$; hence, the amount of blocks administered depended on the participant's pace. Young participants completed on average 15.6 speed blocks and 15.4 accuracy blocks. Older participants completed on average 13.0 speed blocks and 13.5 accuracy blocks.

Preprocessing. The main cells for the analysis of PES contained as many observations as there were errors. To obtain reliable parameter estimates, we selected for analysis only those participants who made at least 50 errors in each condition (speed and accuracy instructions), leaving 15 young and 16 older participants. Furthermore, before carrying out the analyses, we deleted outliers with responses slower than 2,500 ms considered too slow and responses faster than $150 \mathrm{~ms}$ considered too fast.

\section{Results}

Deadlines. As expected, the nondecision times estimated at the beginning of the experimental session were somewhat higher for older (368 ms, $S D=42)$ than for young participants $(350 \mathrm{~ms}$, $S D=28)$. Although this difference is modest on the group level, individualized nondecision time estimates ranged from $265 \mathrm{~ms}$ to $435 \mathrm{~ms}$. This 130-ms range in nondecision time estimates suggests that the deadline adjustment procedure is useful to equate speed pressure over participants.

We first report the descriptive results on RT and accuracy and then turn to the analyses with the EZ-diffusion model.

\footnotetext{
${ }^{1}$ Seniorlab is a organization that mediates between older people and researchers (http://www.seniorlab.nl).
} 

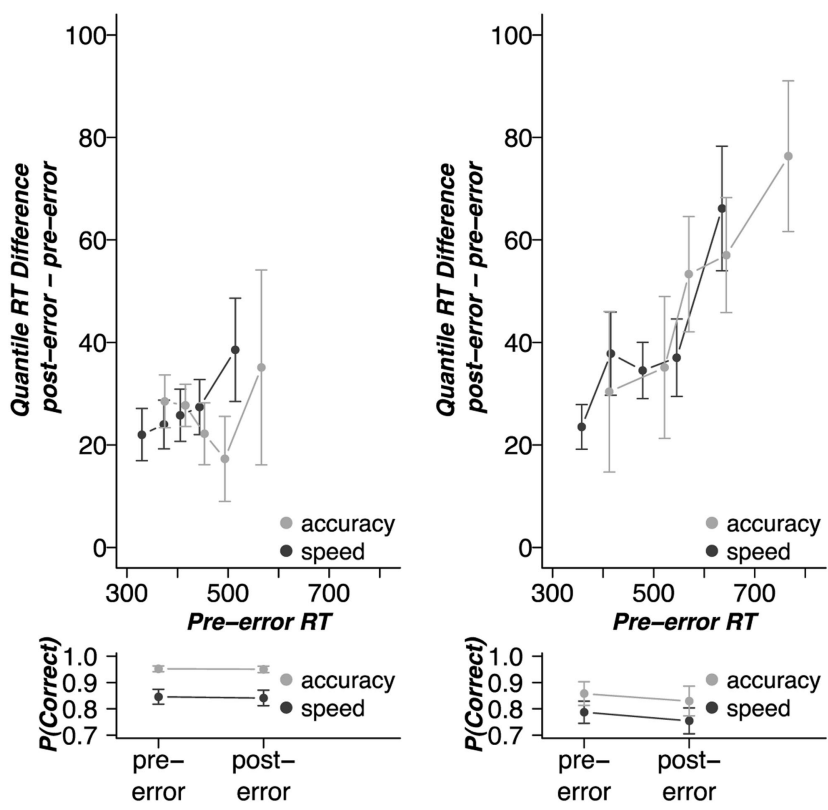

Figure 2. Robust analysis of posterror slowing in Experiment 1 (random dot motion). The figure shows how response time (RT) and accuracy differ between posterror and pre-error trials for both age groups, separately for speed and accuracy blocks. All RT quantiles are slower posterror than pre-error; for older participants, this difference increases over the five RT quantiles (at 10\%, 30\%, 50\%, 70\%, and 90\%). The quantiles on the $x$-axis and differences on the $y$-axis were calculated per participant before averaging. Error bars contain 2 standard errors around across-participant means. Lower panels show that there is no posterror effect on accuracy.

Descriptive results. The current data set illustrates the potential impact of analyzing PES either with the traditional method or with the robust method. In this section, we first report the results of both analyses, beginning with the robust analysis.

Posterror effect on RT and accuracy: Robust analysis. Figure 2 shows the results of the robust analysis, based on a comparison between posterror and pre-error trials. The left panels show results for young participants, and the right panels show results for older participants. The upper panels show the posterror versus pre-error difference for each RT quantile, plotted against the RT quantiles in pre-error trials. This delta plot (De Jong, Liang, \& Lauber, 1994; Pratte, Rouder, Morey, \& Feng, 2010; Speckman, Rouder, Morey, \& Pratte, 2008) shows PES-induced changes across the entire RT distribution.

In both the speed and the accuracy conditions, young participants responded between 20 and $40 \mathrm{~ms}$ slower after an error, a slowdown that is roughly constant across the RT distribution. For older participants, in contrast, the PES effect increased across the RT distribution. In the fast $10 \%$ quantile, older participants were about $30 \mathrm{~ms}$ slower after an error, but this difference increased to around $70 \mathrm{~ms}$ in the slow $90 \%$ quantile. Thus, PES expressed itself differently for young and older participants; for young participants, the RT distribution was shifted by a constant amount, whereas for older participants, errors shifted and skewed the RT distribution. This qualitative difference between young and older participants was only evident from the distribution of RT and would have gone unnoticed had we considered only mean RT.
In both the speed and the accuracy conditions, for both age groups, there appears to be no PES effect on accuracy. Clearly, however, accuracy for both groups was higher in the accuracy condition than in the speed condition.

A comparison of overall performance for older participants and young participants shows that older participants were less accurate than young participants overall. Also, for both the speed and the accuracy condition, the pre-error RT distribution for older participants was shifted and stretched out relative to that for the young participants. This indicates that older participants were slower than young participants over the entire distribution, a difference that increased in the tail of the distribution.

Posterror effect on RT and accuracy: Traditional analysis. Figure 3 shows results based on the traditional analysis of PES, that is, where the $y$-axis represents the traditional quantile RT difference between posterror and postcorrect trials (without the correction discussed above). At first glance, the figure looks similar to Figure 2. However, in Figure 3, the PES effect is smaller in both conditions and for both age groups. Also, Figure 3 suggests that older participants have a PES effect on accuracy. Because we deem these results an artifact of the traditional analysis procedure, we do not discuss them further here.

Now that we have illustrated the importance of contrasting the right conditions, we turn to the diffusion model analysis of the effects found with the robust PES method.
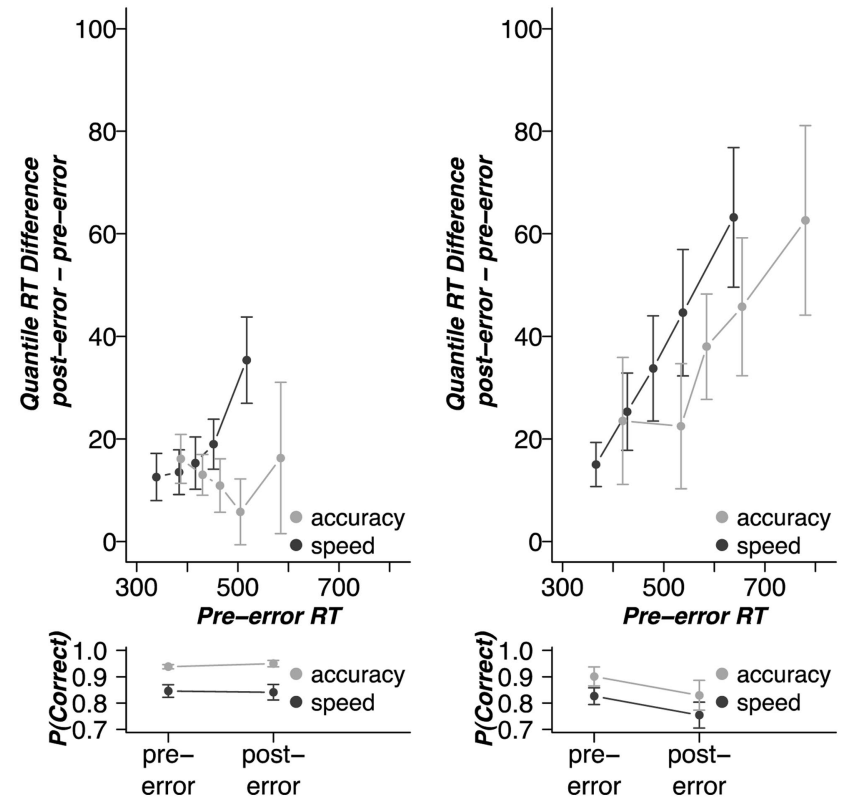

Figure 3. Traditional analysis of posterror slowing in Experiment 1 (random dot motion). The figure shows how response time (RT) and accuracy differ between posterror and postcorrect trials for both age groups, separately for speed and accuracy blocks. All RT quantiles are slower posterror than pre-error; for older participants, this difference increases over the five RT quantiles (at $10 \%, 30 \%, 50 \%, 70 \%$, and $90 \%$ ). The quantiles on the $x$-axis and differences on the $y$-axis were calculated per participant before averaging. Error bars contain 2 standard errors around across-participant means. Lower panels suggest that there is a posterror decrease of accuracy for older participants. The results in this figure serve only as an illustration of the difference between the traditional and robust analysis (see Figure 2). 
Modeling results. Figure 2 shows that the PES effect was qualitatively different for young and older participants, suggesting that different psychological processes underlie the effect for both groups. To quantify this intuition, we applied the EZ-diffusion model and plotted the model parameters in Figure 4. Note that the EZ-diffusion model parameters were calculated separately for each participant. The amount of data available in each cell of the analysis depended on the amount of errors a participant made.

To gauge the difference between parameter values shown in Figure 4, we quantified the evidence in favor of or against the null hypothesis using a default Bayesian $t$ test (Rouder, Speckman, Sun, Morey, \& Iverson, 2009; Wetzels et al., 2011; Wetzels, Raaijmakers, Jakab, \& Wagenmakers, 2009). The resulting Bayes factor $B F_{10}$ quantifies how much more (or less) likely the data are under the alternative hypothesis than under the null hypothesis. For instance, a $B F_{10}$ of 2 indicates that the data are twice as likely under the alternative than under the null hypothesis. An important advantage of calculating the Bayes factor is that it allowed us to quantify evidence in favor of the null as well as in favor of the alternative hypothesis. Analyses were performed on data collapsed over irrelevant conditions.

The two leftmost panels of Figure 4 show that the drift rate was higher for young participants than for older participants $\left(B F_{10}=\right.$ 172.2), indicating that the efficiency of information processing was higher for young than for older participants. The two center panels of Figure 4 show that boundary separation was higher for older participants than for young participants $\left(B F_{10}=417.3\right)$, indicating that older participants were more cautious than young participants. The two rightmost panels of Figure 4 show that nondecision time was higher for older participants than for young participants $\left(B F_{10}=170.4\right)$, indicating that older participants needed more time to encode stimuli and execute the motor response than young participants.
Inspection of the two center panels of Figure 4 also confirms that boundary separation was higher in the accuracy blocks than in the speed blocks for both young $\left(B F_{10}=303.3\right)$ and older $\left(B F_{10}=158.6\right)$ participants.

Note that the error bars in Figure 4 were computed separately for each condition. A more accurate assessment of the PES effects on the model parameters can be obtained by plotting these effects within-subjects, as in Figure 5.

Figure 5 shows, for both young and older participants, the difference in the parameter estimates between pre-error and posterror trials. The posterror effects on neither of the parameters differed between speed and accuracy conditions $\left(B F_{10} \mathrm{~s}<0.5\right)$; therefore, we collapsed the results across the speed and accuracy conditions before plotting the figures and conducting the analyses.

The advantage of Figure 5 over Figure 4 is that it allows an assessment of the distribution of posterror effects over participants. Inspecting the leftmost box plots in each panel from Figure 5, the data show that young participants had a nondecision time that was systematically higher after an error, $B F_{10}=134.7$. Neither drift rate $\left(B F_{10}=0.16\right)$ nor boundary separation $\left(B F_{10}=0.27\right)$ differed before and after errors.

The rightmost box plots in each panel from Figure 5 show the results for older participants. Here, three effects are present. First, older participants had lower drift rates after errors $\left(B F_{10}=\right.$ 1872.9), indicating a decrease in the speed of information processing. Second, boundary separation increased after errors $\left(B F_{10}=\right.$ 10.3), indicating an increase in response caution. Finally, as for young participants, nondecision times increased after errors $\left(B F_{10}=16.7\right)$, indicating that older participants might waste some time on irrelevant processes after committing an error.

Table 1 shows the across-participant correlations between posterror effects on the three parameters. The correlations suggest that for both young and older participants, the effects on drift rate and

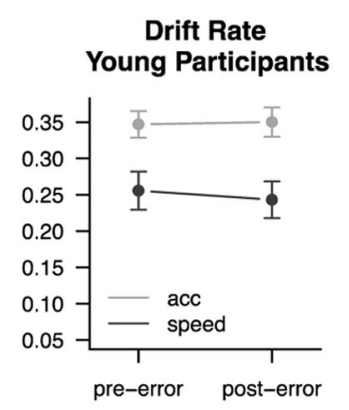

Drift Rate Elderly Participants

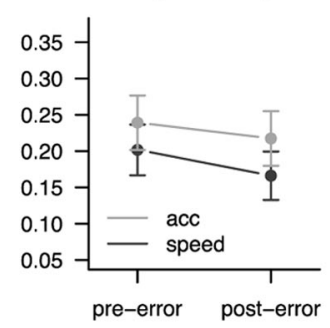

\section{Boundary Separation} Young Participants

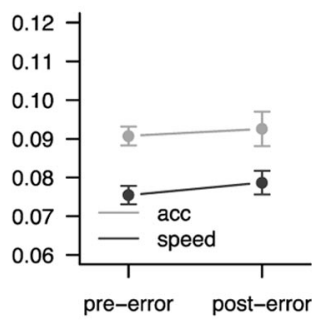

Boundary Separation Elderly Participants

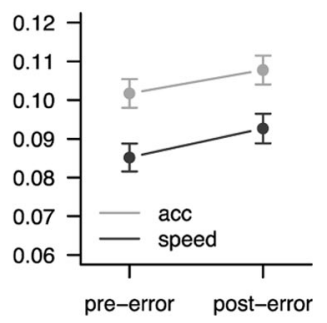

Non-Decision Time Young Participants

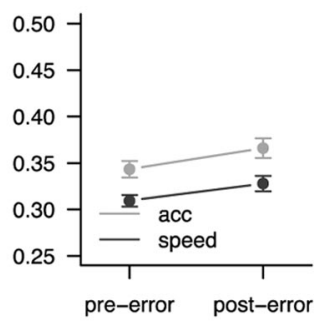

Non-Decision Time Elderly Participants

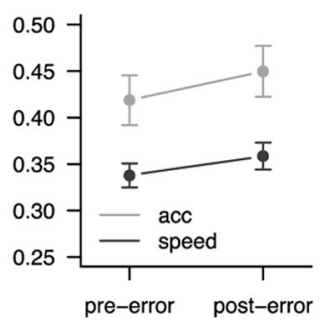

Figure 4. Experiment 1 (random dot motion) posterror effects on the three parameters of the EZ-diffusion model separately for the two age groups and the two experimental conditions. Error bars enclose 2 standard errors. See text for details. 

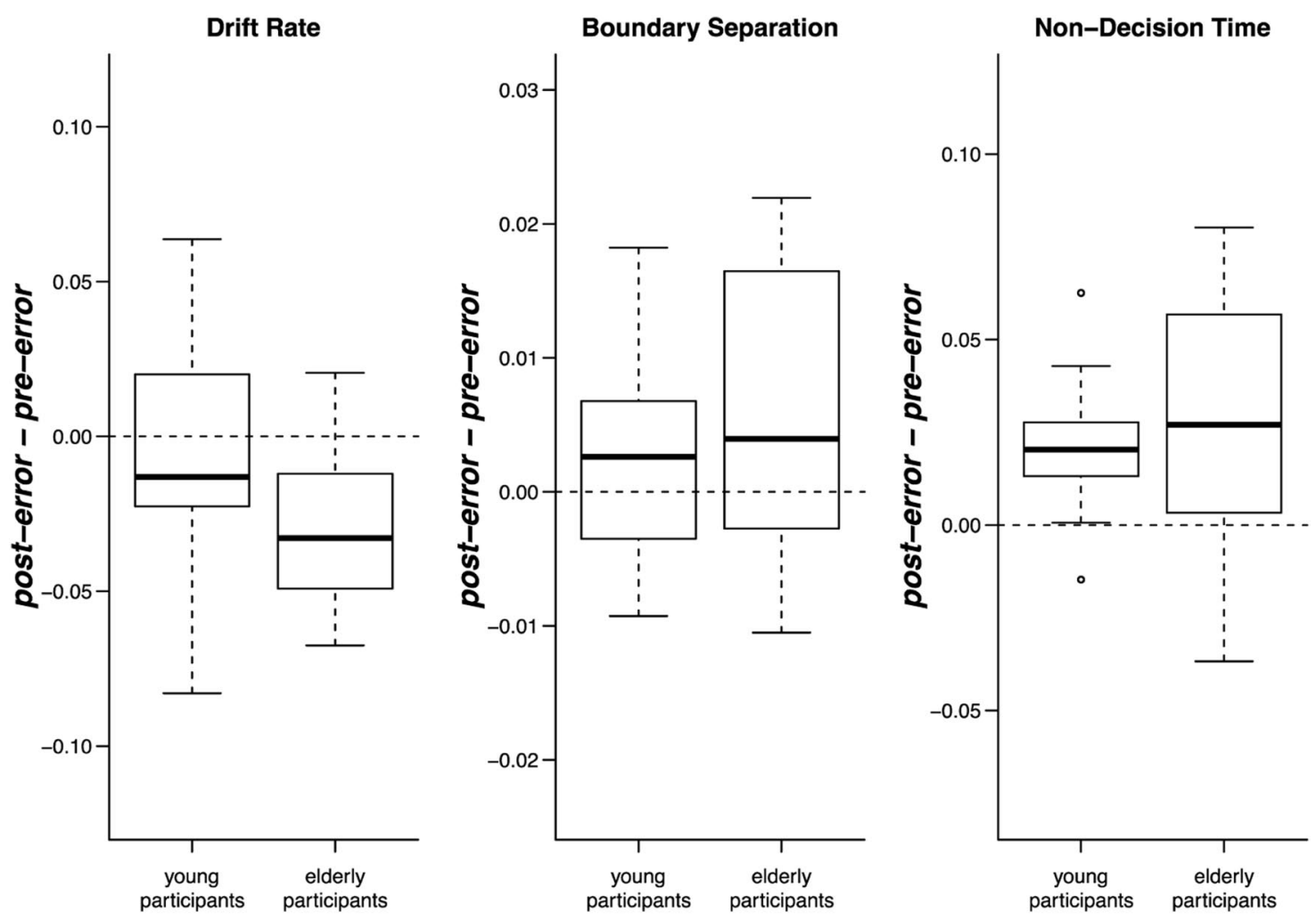

Figure 5. Experiment 1. Box plots showing the posterror effects (posterror - pre-error) on the three EZ-diffusion parameters for both young and older participants, collapsed over speed and accuracy conditions. The boxes represent the .25 and .75 percentiles of the distribution of parameter effects. The error bars extend to the highest and lowest value, excluding the values that are away from the box' outer edge more than 1.5 times the quartile range within the box. Those extreme values are plotted as separate points.

boundary often go hand in hand. Also, for older participants, the effects on drift rate are often accompanied by effects on nondecision time. These correlations suggest that the multivariate nature of the posterror effect that is shown in Figures 4 and 5 cannot be attributed solely to the aggregation of different univariate effects over participants.

Table 1

Correlations Between Parameters in Experiment 1 (Random Dot Motion)

\begin{tabular}{cccc}
\hline Group & $\Delta v$ & $\Delta a$ & $\Delta T_{e r}$ \\
\hline Young & & & \\
$\Delta v$ & - & $B F_{10}>1000$ & $B F_{10}=1.3$ \\
$\Delta a$ & $-\mathbf{8 9}$ & - & $B F_{10}=2.7$ \\
$\Delta T_{e r}$ & $\mathbf{4 7}$ & -.56 & - \\
Older & & & $B F_{10}=14.9$ \\
$\Delta v$ & - & -.61 & $B F_{10}=4.6$ \\
$\Delta a$ & -.79 & $\mathbf{. 6 1}$ & - \\
$\Delta T_{e r}$ & $\mathbf{7 0}$ & & \\
\hline
\end{tabular}

Note. $\quad v=$ drift rate; $a=$ boundary separation; $T_{e r}=$ nondecision time. Across-participant correlations between parameter effects are shown in the lower left triangles (bold face). The upper right triangles contain the associated two-sided Bayes factors $\left(B F_{10}\right)$ in favor of the alternative hypothesis of a nonzero correlation.
The above results are statistically compelling: The Bayes factors clearly indicate that, for older participants, posterror effects are reflected in drift rate, boundary separation, and nondecision time; for young participants, the Bayes factors clearly indicate that posterror effects are reflected in nondecision time. Compared with $p$ values, Bayes factors are more conservative and less eager to support the alternative hypothesis (e.g., Berger \& Sellke, 1987; Wetzels et al., 2011). For example, data for which the $p$ value equals .05 yield at most a Bayes factor of 2.46, and data for which the $p$ value equals .01 yield at most a Bayes factor of 7.99 (Sellke, Bayarri, \& Berger, 2001). ${ }^{2}$ In addition, the default Bayesian $t$ test is relatively conservative. Taken together, this means that the evidence for the above effects is relatively strong.

Moreover, our conclusions are conceptually consistent with the observed data: Following an error, the RT distributions for older participants skew out, and this is accommodated by a decrease in drift rate. At the same time, boundary separation has to increase in order to keep accuracy constant.

A note of caution: Our statistical analyses do not warrant the claim that, for young participants, posterror effects are reflected

\footnotetext{
${ }^{2}$ These Bayes factors are upper bounds obtained by considering the data and choosing the parameter prior that provides maximum support for the alternative hypothesis. See Sellke et al. (2001) for details.
} 
only in nondecision time. This claim requires that the Bayes factor supports the null hypothesis of no error-induced effects on drift rate and boundary separation. For the present data set, the evidence in favor of both null hypotheses is suggestive but not conclusive. This ambiguity is also apparent from the fact that the error-induced effects on drift rate and boundary separation are not statistically different between young and older participants (i.e., $B F_{10}=1.41$ and $B F_{10}=0.26$, respectively). Hence, it would be premature to conclude that the PES effects on the model parameters are different between the young and the older participants (Gelman \& Stern, 2006; Nieuwenhuis, Forstmann, \& Wagenmakers, 2011). Nevertheless, the data do warrant the conclusion that for older participants, PES manifests itself in drift rate, boundary separation, and nondecision time, whereas for young participants, PES manifests itself in nondecision time.

Model evaluation. Figure 6 shows the empirical quantiles for all experimental conditions plotted against the diffusion model predictions based on the parameter values displayed in Figure 4. The fit is very satisfactory in general, although in the accuracy blocks for older participant, there is a slight misfit in the highest $(90 \%)$ quantile for both pre-error and posterror trials.

\section{Experiment 2: Lexical Decision}

In Experiment 1, the posterror effects for young and older participants were contrasted in a moving dots task. The locus of these posterror effects might be specific to the domain of perceptual decision making. In addition, the posterror effects were modest in size, perhaps reflecting a limited error awareness or a realization that the perceptual errors are to some extent unavoidable. For these reasons, it is important to assess the generalizability of the results obtained in Experiment 1. To this aim, we now present data from a very different decision-making task: lexical decision. This data set has been published previously by Ratcliff et al. (2004), but the posterror effects in this data set remain to be examined.

\section{Method}

The data that we analyze below comprise the two lexical decision experiments of Ratcliff et al. (2004). In both studies, young and older participants responded to 2,100 lexical decision stimuli. The two experiments differed only with respect to the nonword stimuli used. In the first experiment, the nonwords were pronounceable; in the second experiment, the nonwords were random letter strings. Preliminary analyses showed that the posterror effects in both data sets were very similar. So, for ease of presentation, we report the results of both data sets combined. The first data set in Ratcliff et al. (2004) comprises 54 young and 44 older participants; the second comprises 54 different young and 40 different older participants.

The analyses presented below are again based on posterror versus pre-error trials. The number of errors that each participant made thus limits the number of data points. Again, we therefore performed analyses on only those participants who committed at least 50 errors. This selection left 102 young and 58 older participants' data to analyze. As in Experiment 1, we deleted responses slower than 2,500 $\mathrm{ms}$ and responses faster than $150 \mathrm{~ms}$.
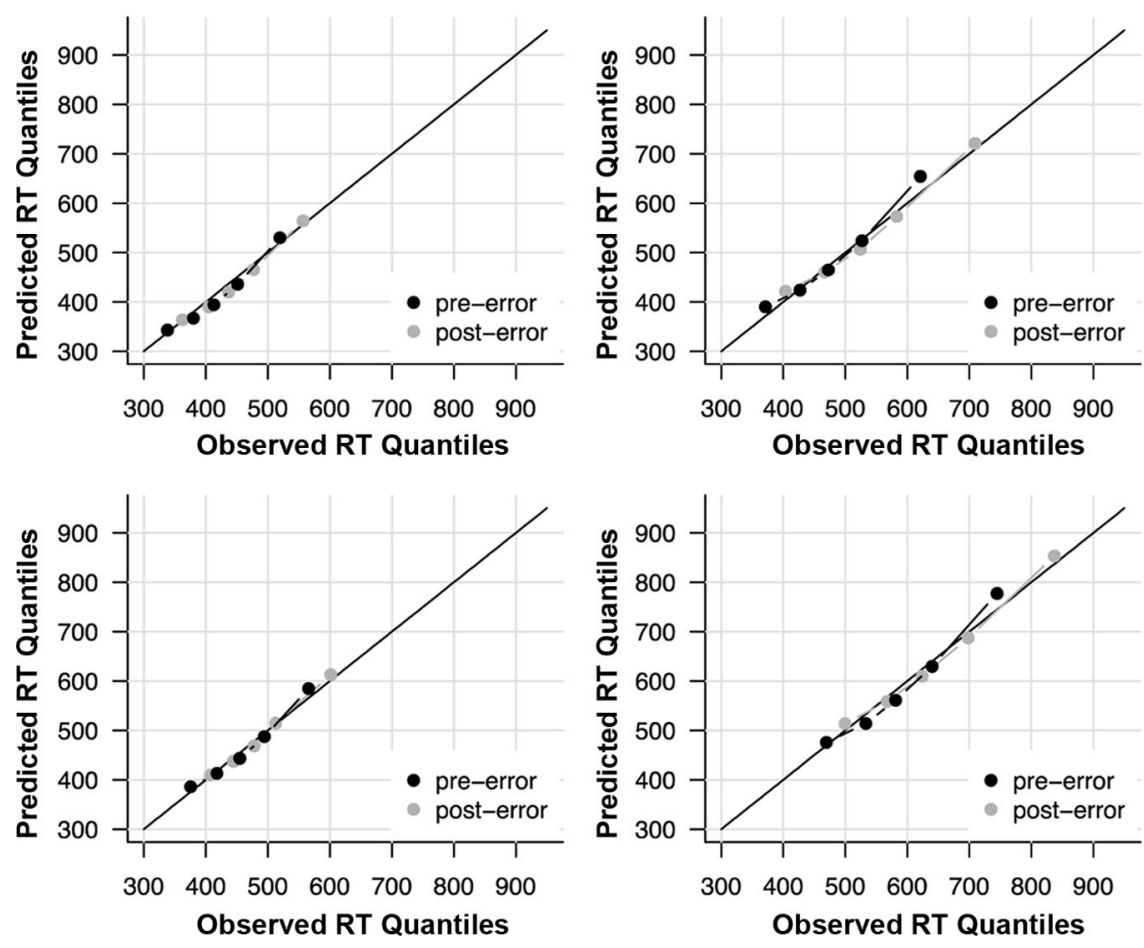

Figure 6. Model fit for the data from Experiment 1 (random dot motion). Empirical response time (RT) quantiles $(10 \%, 30 \%, 50 \%, 70 \%$, and $90 \%)$ are plotted against RT quantiles predicted by the EZ-diffusion model. 


\section{Results}

Descriptive results. The upper panels of Figure 7 display the effect of errors on RT as a delta plot (compare Figure 2). The effects of errors on RT are clearly much larger than the effects in the perceptual task of Experiment 1. The upper left panel shows that young participants were significantly slower after errors than before errors. In particular, the effect on the slow $90 \%$ quantile of the RT distribution (about $320 \mathrm{~ms}$ ) was larger than the effect on the fast $10 \%$ quantile of the distribution (about $80 \mathrm{~ms}$ ). The right panel shows that the effects for older participants were much stronger than the effects for younger participants. Again, the effect of errors was much larger in the tail of the distribution (about $590 \mathrm{~ms}$ ) than in the leading edge of the distribution (about $200 \mathrm{~ms}$ ). The lower two panels show that errors had no effect on the accuracy of responses for either young or older participants.

Modeling results. The modeling of the data of Experiment 2 was performed in the same way as the modeling in Experiment 1. The sole difference between the two situations is that Experiment 2 did not feature a speed-accuracy manipulation. The three panels of Figure 8 each show a parameter of the EZ-diffusion model before and after errors for both young and older participants. Drift rate did not differ between young and older participants $\left(B F_{10}=\right.$ 0.089). Boundary separation and nondecision time were higher for older than for young participants $\left(B F_{10} \geq 1000\right.$ for both parame-
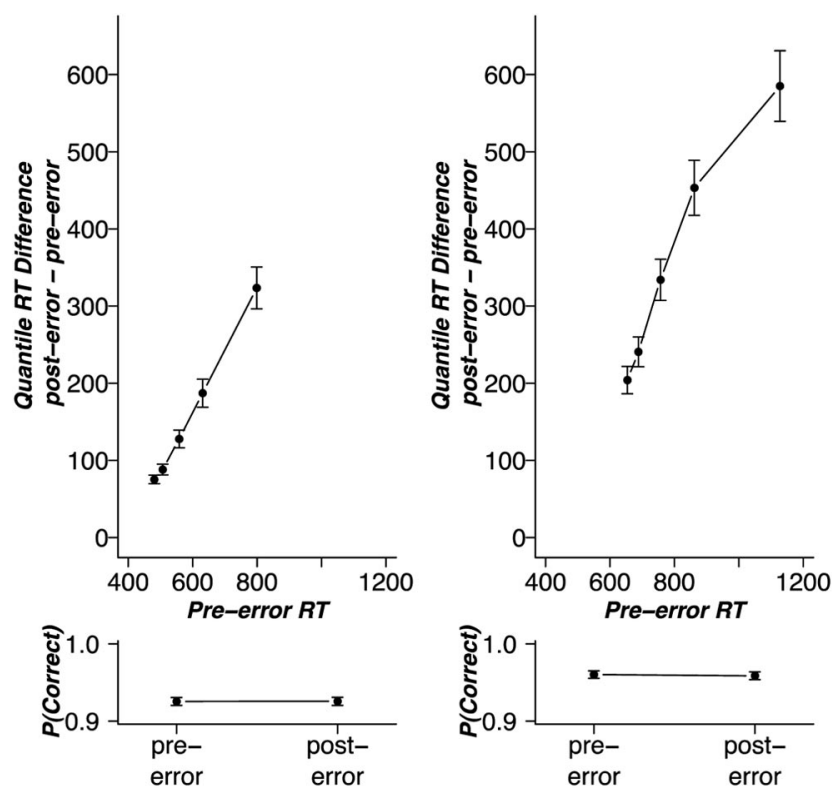

Figure 7. Robust analysis of posterror slowing in Experiment 2 (lexical decision). The figure shows how correct response time (RT) and accuracy differ between posterror and pre-error trials for both age groups, separately for speed and accuracy blocks. All RT quantiles are slower posterror than pre-error; for both young and older participants, this difference increases over the five RT quantiles (at 10\%, 30\%, 50\%, 70\%, and 90\%). The quantiles on the $x$-axis (at 10\%, 30\%, 50\%, 70\%, and 90\%) and differences on the $y$-axis were calculated per participant before averaging. Error bars contain 2 standard errors around across-participant means. Lower panels show that there is no posterror effect on accuracy for either young or older participants. ters). These results qualitatively replicate the results of Ratcliff et al. (2004).

The distributions of within-participant differences in parameter estimates between pre-error and posterror trials are shown in Figure 9. The leftmost box plots in each panel from Figure 9 show the results for young participants. First, young participants had lower drift rates after errors $\left(B F_{10} \geq 1000\right)$, indicating a decreased speed of information processing. Second, young participants had a higher boundary separation $\left(B F_{10} \geq 1000\right)$ after errors, indicating an increased response caution. No posterror effect was found on nondecision time $\left(B F_{10}=0.25\right)$.

The rightmost box plots in each panel show the results for older participants. First, older participants had lower drift rates $\left(B F_{10} \geq\right.$ 1000) after errors, indicating a decreased speed of information processing. Second, they showed higher boundary separation $\left(B F_{10} \geq 1000\right)$, indicating increased response caution. Third, older participants had a higher nondecision time after errors $\left(B F_{10} \geq\right.$ 1000), indicating that those participants spent time on irrelevant processes after committing an error.

A test on the age differences for the posterror effects confirmed that the only posterror effect to differ between young and older participants was the one for nondecision time $\left(B F_{10} \geq 1000\right)$, that is, after an error the older participants increased their nondecision time more than younger participants. Table 2 shows the correlations between posterror effects on the three parameters of the EZ-diffusion model. The positive correlations suggest that the multivariate nature of the posterror effect in Figures 8 and 9 was not caused by aggregating different univariate effects over participants.

Model evaluation. Figure 10 shows the empirical quantiles for all experimental conditions plotted against the diffusion model predictions based on the parameter values displayed in Figure 8. The fit is very satisfactory in general, although the lowest quantiles for both young and older participants are slightly underestimated for both pre-error and posterror responses.

\section{Discussion}

This study focused on performance differences between young and older participants in speeded decision-making tasks. Our results confirmed that older participants are more cautious than young participants. Also, our results confirmed that nondecision times are larger for older participants and that, only in perceptual tasks, the speed of information processing tends to be lower for older participants (Ratcliff et al., 2006a, 2006b; but see Spaniol et al., 2006). More specifically, however, the goal of this study was to study age-related differences in posterror performance. Our PES effects are consistent with those reported by Smith and Brewer (1995): Older participants slow down more strongly after errors than young participants. A delta plot of the data revealed that in a perceptual task, the PES effect for young participants was qualitatively different from that for older participants; for young individuals, an error only shifts the RT distribution, whereas for older individuals, an error shifts and skews the RT distribution. In the lexical decision task, the PES effect was qualitatively similar for both age groups, but more pronounced for the older participants.

To obtain a more detailed understanding of the psychological processes that drive the posterror effects, we applied the diffu- 


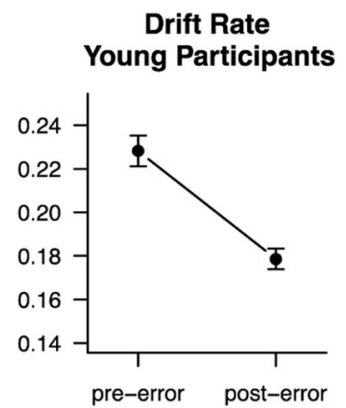

Drift Rate Elderly Participants

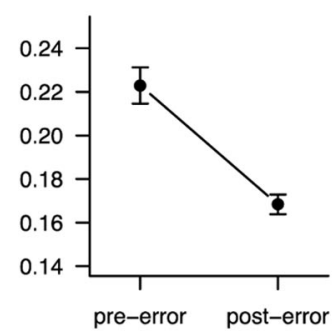

Boundary Separation Young Participants

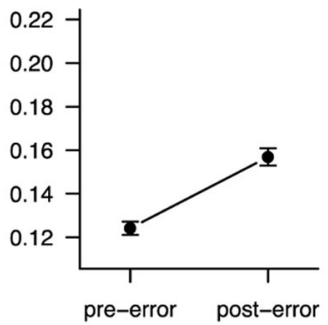

Boundary Separation Elderly Participants

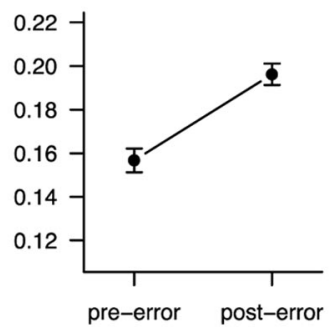

Non-Decision Time Young Participants

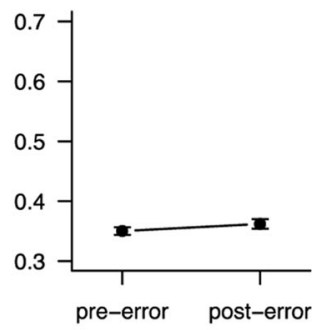

Non-Decision Time Elderly Participants

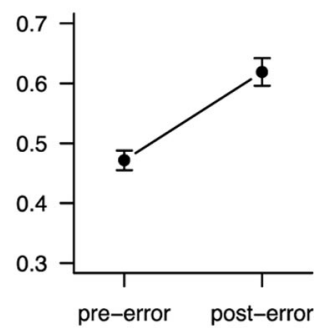

Figure 8. Experiment 2 (lexical decision) posterror effects on the three parameters of the EZ-diffusion model separately for the two age groups. Error bars enclose 2 standard errors. See text for details.

sion model to the observed data. The diffusion model decomposition showed that nondecision time increased after an error for both young and older participants in the perceptual task and only for the older participants in the lexical decision task. This change in the shift of the RT distribution can have several reasons, but two hypotheses seem particularly plausible in these experiments. First, it is possible that participants needed time to evaluate the error, to overcome disappointment, or to internally berate themselves. Second, it is possible that on the trial following an error, participants pressed the response button less firmly because of error-induced hesitation or lack of confidence. The diffusion model cannot distinguish between these hypotheses because the nondecision time encompasses both the time prior to the onset of the information accumulation process and the time after the information accumulation process has terminated (see Figure 1). Nevertheless, future work could test the hesitation hypothesis empirically by using a membrane key or force button; if the hesitation hypothesis is true, a more sensitive measurement of response execution may reduce or eliminate the posterror effect on nondecision time. The young college students in Experiment 2 differed from the older participants in that they did not show a posterror increase in nondecision time. This difference might reflect the college students' experience with the lexical decision task. This experience might have caused indifference concerning the errors that are inevitably committed in performing such a task. Therefore, no time was spent thinking over the errors.

The diffusion model decomposition also revealed that, for the older participants in the perceptual task and for both young and older participants in the lexical decision task, the occurrence of an error reduced drift rate. A plausible explanation for this reduction in the efficiency of information processing is that the evaluation of the error message is still ongoing when the next stimulus has to be processed; as a result, fewer cognitive resources are available for the primary task.

Finally, the diffusion model decomposition showed that older participants in the perceptual task and both young and older participants in the lexical decision task tended to become more cautious after committing an error. This finding is in line with the common explanation of PES as a strategic adjustment of response thresholds, and it is consistent with conclusions drawn by Smith and Brewer (1995) and Dutilh, Vandekerckhove, et al. (2012). By increasing response thresholds, participants are able to increase the probability of a correct response at the cost of a decrease in response speed.

Altogether, the results from this study challenge the common explanation of PES in terms of an adjustment in boundary separation. In contrast to this unitary explanation, PES appears to be a multifaceted phenomenon that is associated with changes in three psychological processes. Furthermore, the comparison of the two different tasks in this study showed that the nature of PES depends on both individual differences (viz., age) and task settings (viz., perceptual vs. lexical decision making). For example, consider the finding that young participants in the perceptual task did not increase their response caution, whereas they did in the lexical decision task. A plausible explanation for this difference might be that in a lexical decision task, participants are usually aware of their errors; moreover, participants may realize that if they had responded more carefully, they could have avoided most errors. In the moving dots task, in contrast, participants may only have limited error awareness; moreover, they may not feel that responding more carefully reduces the probability of making an error. This suggestion is supported by the results of Dutilh, Vande- 

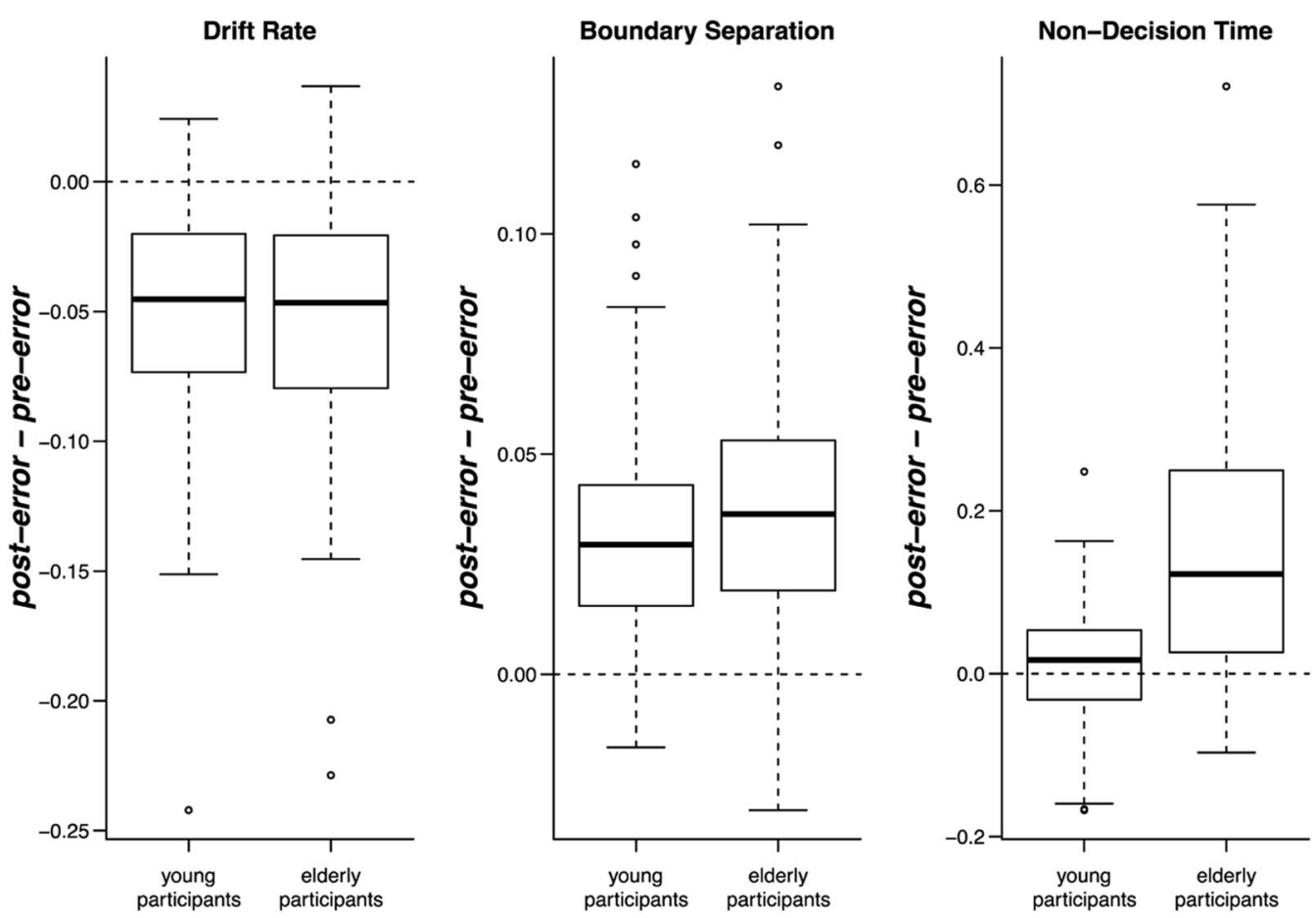

Figure 9. Experiment 2 (lexical decision) box plots showing the posterror effects (posterror - pre-error) on the three EZ-diffusion parameters for both young and older participants. The boxes represent the .25 and .75 percentiles of the distribution of parameter effects. The error bars extend to the highest and lowest value, excluding the values that are away from the box' outer edge more than 1.5 times the quartile range within the box. Those extreme values are plotted as separate points.

kerckhove, et al. (2012), who applied the diffusion model to assess and decompose PES in a large lexical decision experiment. Dutilh et al. concluded that in this lexical decision experiment, PES was largely due to an increase in response caution after errors. In sum, these different results suggest that the components that underlie the PES effect depend on factors that vary across tasks and participants. Moreover, the different components of the PES effect might be mediated by common underlying factors, such as error awareness and the negative

Table 2

Correlations Between Parameters in Experiment 2 (Lexical Decision)

\begin{tabular}{cccc}
\hline Group & $\Delta v$ & $\Delta a$ & $\Delta T_{e r}$ \\
\hline Young & & & \\
$\Delta v$ & - & $B F_{10}>1000$ & $B F_{10}>1000$ \\
$\Delta a$ & $-\mathbf{7 5}$ & -.29 & $B F_{10}=10.4$ \\
$\Delta T_{e r}$ & $\mathbf{4 5}$ & & - \\
Older & - & $B F_{10}>1000$ & $B F_{10}=7.6$ \\
$\Delta v$ & $-\mathbf{8 5}$ & -.36 & $B F_{10}=7.7$ \\
$\Delta a$ & $\mathbf{3 6}$ & -.36 & - \\
$\Delta T_{e r}$ &
\end{tabular}

Note. $\quad v=$ drift rate; $a=$ boundary separation; $T_{e r}=$ nondecision time. Across-participant correlations between parameter effects are shown in the lower left triangles (bold face). The upper right triangles contain the associated two-sided Bayes factors $\left(B F_{10}\right)$ in favor of the alternative hypothesis of a nonzero correlation. affect associated with errors. Future experimental work should establish how error awareness and the affective consequences of errors influence the nature of the PES effect.

The conclusions in this study were made possible by a combination of two relatively novel methods in the analysis of PES. First was the application of a robust measure for PES (i.e., $R T_{\text {posterror }}-$ $R T_{\text {pre-error }}$ ) that is not confounded by global changes in motivation or response caution in the way that the traditional measure (i.e., $R T_{\text {posterror }}-R T_{\text {postcorrect }}$ ) is. Second, the application of the diffusion model allowed us to study not just changes in RT and accuracy, but draw conclusions based on the underlying psychological processes that change following an error.
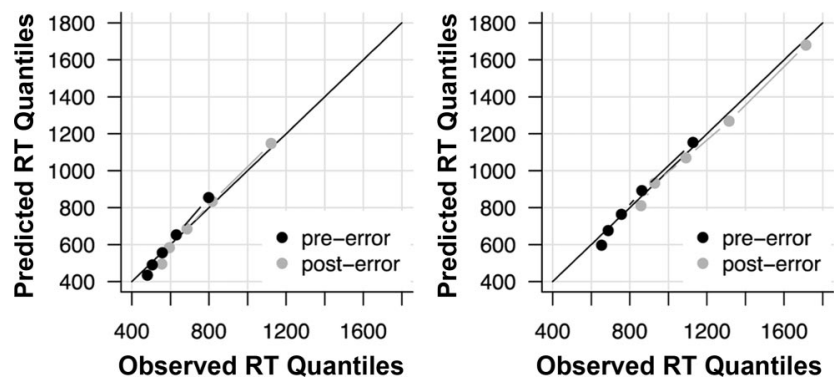

Figure 10. Model fit for the data from Experiment 2 (lexical decision). Empirical RT quantiles $(10 \%, 30 \%, 50 \%, 70 \%$, and $90 \%)$ are plotted against response time (RT) quantiles predicted by the EZ-diffusion model. 


\section{References}

Band, G. P. H., \& Kok, A. (2000). Age effects on response monitoring in a mental-rotation task. Biological Psychology, 51, 201-221.

Berger, J. O., \& Sellke, T. (1987). Testing a point null hypothesis: The irreconcilability of $\mathrm{p}$ values and evidence. Journal of the American Statistical Association, 82, 112-139.

Brinley, J. F. (1965). Cognitive sets, speed and accuracy of performance in the elderly. In A. T. Welford \& J. E. Birren (Eds.), Behavior, aging and the nervous system (pp. 114-149). Springfield, IL: Thomas.

Britten, K. H., Shadlen, M. N., Newsome, W. T., \& Movshon, J. A. (1992). The analysis of visual motion: A comparison of neuronal and psychophysical performance. The Journal of Neuroscience, 12, 4745-4765.

Cerella, J. (1985). Information processing rates in the elderly. Psychological Bulletin, 98, 67-83.

Danielmeier, C., \& Ullsperger, M. (2011). Post-error adjustments. Frontiers in Psychology, 2, 233. doi:10.3389/fpsyg.2011.00233

De Jong, R., Liang, C.-C., \& Lauber, E. (1994). Conditional and unconditional automaticity: A dual-process model of effects of spatial stimulus-response correspondence. Journal of Experimental Psychology: Human Perception and Performance, 20, 731-750.

Dutilh, G., Vandekerckhove, J., Forstmann, B. U., \& Wagenmakers, E.-J. (2012). Testing theories of post-error slowing. Attention, Perception, \& Psychophysics, 74, 454-465.

Dutilh, G., van Ravenzwaaij, D., Nieuwenhuis, S., Van der Maas, H. L. J., Forstmann, B. U. D., \& Wagenmakers, E.-J. (2012). How to measure post-error slowing: A confound and a simple solution. Journal of Mathematical Psychology, 56, 208-216.

Gelman, A., \& Stern, H. (2006). The difference between "significant" and "not significant" is not itself statistically significant. The American Statistician, 60, 328-331.

Kamienkowski, J. E., Pashler, H., Dehaene, S., \& Sigman, M. (2011). Effects of practice on task architecture: Combined evidence from interference experiments and random-walk models of decision making. Cognition, 119, 81-95.

Laming, D. (1968). Information theory of choice-reaction times. New York, NY: Academic Press.

Laming, D. (1979a). Autocorrelation of choice-reaction times. Acta Psychologica, 43, 381-412.

Laming, D. (1979b). Choice reaction performance following an error. Acta Psychologica, 43, 199-224.

Mata, R., \& Nunes, L. (2010). When less is enough: Cognitive aging, information search, and decision quality in consumer choice. Psychology and Aging, 25, 289-298

Nieuwenhuis, S., Forstmann, B. U., \& Wagenmakers, E.-J. (2011). Erroneous analyses of interactions in neuroscience: A problem of significance. Nature Neuroscience, 14, 1105-1107.

Notebaert, W., Houtman, F., Opstal, F., Gevers, W., Fias, W., \& Verguts, T. (2009). Post-error slowing: An orienting account. Cognition, 111, 275-279.

Pratte, M. S., Rouder, J. N., Morey, R. D., \& Feng, C. (2010). Exploring the differences in distributional properties between Stroop and Simon effects using delta plots. Attention, Perception, \& Psychophysics, 72, 2013-2025.

Rabbitt, P. (1979). How old and young subjects monitor and control responses for accuracy and speed. British Journal of Psychology, 70, 305-311.

Rabbitt, P., \& Rodgers, B. (1977). What does a man do after an error? An analysis of response programming. Quarterly Journal of Experimental Psychology, 29, 727-743.

Rabbitt, P., \& Vyas, S. M. (1980). Selective anticipation for events in old age. Journal of Gerontology, 35, 913-919.

Ratcliff, R. (1978). A theory of memory retrieval. Psychological Review, $85,59-108$.
Ratcliff, R., \& McKoon, G. (2008). The diffusion decision model: Theory and data for two-choice decision tasks. Neural Computation, 20, 873922.

Ratcliff, R., Spieler, D., \& McKoon, G. (2000). Explicitly modeling the effects of aging on response time. Psychonomic Bulletin \& Review, 7, $1-25$.

Ratcliff, R., Thapar, J., \& McKoon, G. (2004). A diffusion model analysis of the effects of aging in the lexical-decision task. Psychology and Aging, 19, 278-289.

Ratcliff, R., Thapar, J., \& McKoon, G. (2006a). Aging and individual differences in rapid two-choice decisions. Psychonomic Bulletin \& Review, 13, 626-635.

Ratcliff, R., Thapar, J., \& McKoon, G. (2006b). Aging, practice, and perceptual tasks: A diffusion model analysis. Psychology and Aging, 21, 353-371.

Ratcliff, R., Thapar, J., \& McKoon, G. (2010). Individual differences, aging, and IQ in two-choice tasks. Cognitive Psychology, 60, 127-157.

Ratcliff, R., \& Tuerlinckx, F. (2002). Estimating parameters of the diffusion model: Approaches to dealing with contaminant reaction times and parameter variability. Psychological Bulletin \& Review, 9, 438-481.

Rouder, J. N., Speckman, P. L., Sun, D., Morey, R. D., \& Iverson, G. (2009). Bayesian $t$ tests for accepting and rejecting the null hypothesis. Psychonomic Bulletin \& Review, 16, 225-237.

Salthouse, T. A. (1979). Adult age and the speed-accuracy trade-off. Ergonomics, 22, 811-821.

Salthouse, T. A. (1985). A theory of cognitive aging. Amsterdam, Netherlands: Elsevier.

Salthouse, T. A. (1996). The processing-speed theory of adult age differences in cognition. Psychological Review, 103, 403-428.

Schmiedek, F., Lövden, M., \& Lindenberger, U. (2009). On the relation of mean reaction time and intraindividual reaction time variability. Psychology and Aging, 24, 841-857.

Schmiedek, F., Oberauer, K., Wilhelm, O., Suss, H.-M., \& Wittmann, W. W. (2007). Individual differences in components of reaction time distributions and their relations to working memory and intelligence. Journal of Experimental Psychology: General, 136, 414-429.

Sellke, T., Bayarri, M. J., \& Berger, J. O. (2001). Calibration of p values for testing precise null hypotheses. The American Statistician, 55, 62 71

Smith, G. A., \& Brewer, N. (1995). Slowness and age: Speed-accuracy mechanisms. Psychology and Aging, 10, 238-247.

Spaniol, J., Madden, D. J., \& Voss, A. (2006). A diffusion model analysis of adult age differences in episodic and semantic long-term memory retrieval. Journal of Experimental Psychology: Learning, Memory, and Cognition, 32, 101-117.

Speckman, P. L., Rouder, J. N., Morey, R. D., \& Pratte, M. S. (2008). Delta plots and coherent distribution ordering. The American Statistician, 62, 262-266.

Starns, J. J., \& Ratcliff, R. (2010). The effects of aging on the speedaccuracy compromise: Boundary optimality in the diffusion model. Psychology and Aging, 25, 377-390.

Strayer, D. L., Wickens, C. D., \& Braune, R. (1987). Adult age differences in the speed and capacity of information processing: II. An electrophysiological approach. Psychology and Aging, 2, 99-110.

Thapar, A., Ratcliff, R., \& McKoon, G. (2003). A diffusion model analysis of the effects of aging on letter discrimination. Psychology and Aging, $18,415-429$.

van Ravenzwaaij, D., Dutilh, G., \& Wagenmakers, E.-J. (2012). A diffusion model decomposition of the effects of alcohol on perceptual decision making. Psychopharmacology, 219, 1017-1025.

van Ravenzwaaij, D., \& Oberauer, K. (2009). How to use the diffusion model: Parameter recovery of three methods: EZ, fast-dm, and DMAT. Journal of Mathematical Psychology, 53, 463-473. 
Wagenmakers, E.-J. (2009). Methodological and empirical developments for the Ratcliff diffusion model of response times and accuracy. European Journal of Cognitive Psychology, 21, 641-671.

Wagenmakers, E.-J., Van der Maas, H. L. J., \& Grasman, R. P. P. P. (2007). An EZ-diffusion model for response time and accuracy. Psychonomic Bulletin \& Review, 14, 3-22.

Wetzels, R., Matzke, D., Lee, M. D., Rouder, J. N., Iverson, G. J., \& Wagenmakers, E.-J. (2011). Statistical evidence in experimental psychology: An empirical comparison using $855 \mathrm{t}$ tests. Perspectives on Psychological Science, 6, 291-298.

Wetzels, R., Raaijmakers, J. G. W., Jakab, E., \& Wagenmakers, E.-J. (2009). How to quantify support for and against the null hypothesis: A flexible WinBUGS implementation of a default Bayesian t test. Psychonomic Bulletin \& Review, 16, 752-760.

White, C. N., Ratcliff, R., Vasey, M. W., \& McKoon, G. (2010). Using diffusion models to understand clinical disorders. Journal of Mathematical Psychology, 54, 39-52.

Wickelgren, W. A. (1977). Speed-accuracy tradeoff and information processing dynamics. Acta Psychologica, 41, 67-85.

Received August 29, 2011

Revision received June 11, 2012

Accepted June 14, 2012

\section{Members of Underrepresented Groups: Reviewers for Journal Manuscripts Wanted}

If you are interested in reviewing manuscripts for APA journals, the APA Publications and Communications Board would like to invite your participation. Manuscript reviewers are vital to the publications process. As a reviewer, you would gain valuable experience in publishing. The P\&C Board is particularly interested in encouraging members of underrepresented groups to participate more in this process.

If you are interested in reviewing manuscripts, please write APA Journals at Reviewers@apa.org. Please note the following important points:

- To be selected as a reviewer, you must have published articles in peer-reviewed journals. The experience of publishing provides a reviewer with the basis for preparing a thorough, objective review.

- To be selected, it is critical to be a regular reader of the five to six empirical journals that are most central to the area or journal for which you would like to review. Current knowledge of recently published research provides a reviewer with the knowledge base to evaluate a new submission within the context of existing research.

- To select the appropriate reviewers for each manuscript, the editor needs detailed information. Please include with your letter your vita. In the letter, please identify which APA journal(s) you are interested in, and describe your area of expertise. Be as specific as possible. For example, "social psychology" is not sufficient-you would need to specify "social cognition" or "attitude change" as well.

- Reviewing a manuscript takes time (1-4 hours per manuscript reviewed). If you are selected to review a manuscript, be prepared to invest the necessary time to evaluate the manuscript thoroughly.

APA now has an online video course that provides guidance in reviewing manuscripts. To learn more about the course and to access the video, visit http://www.apa.org/pubs/authors/reviewmanuscript-ce-video.aspx. 